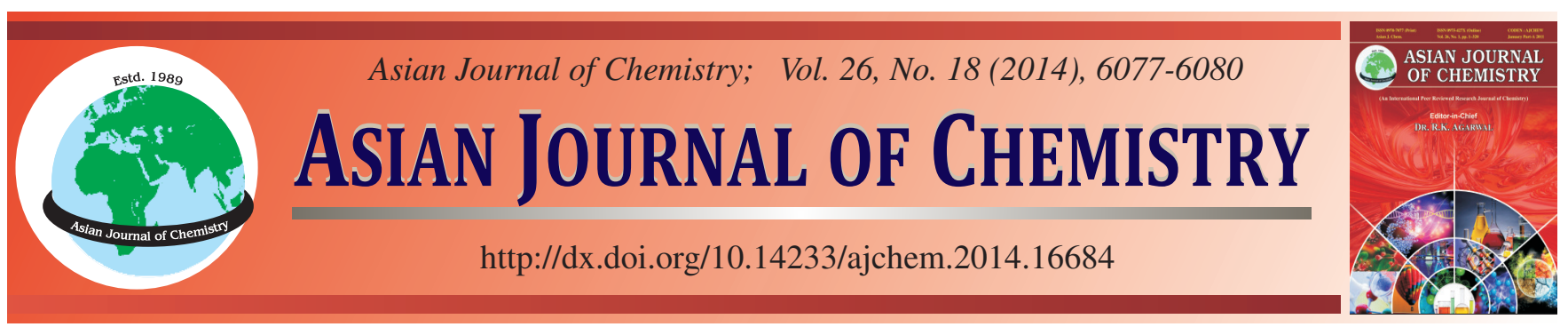

\title{
Enhanced Control on the Electro Deposition Through Magnetic Field Using Reverse Microemulsion as Template
}

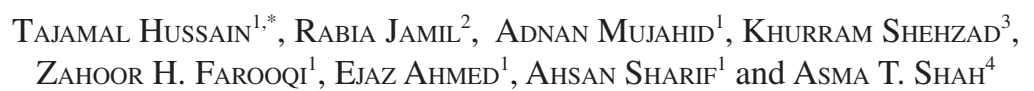

${ }^{1}$ Institute of Chemistry, University of the Punjab, Lahore-54590, Pakistan

${ }^{2}$ Department of Chemistry, Quaid-i-Azam University, Islamabad-45320, Pakistan

${ }^{3}$ Center for Nano and Micro Mechanics, Tsinghua University, Beijing 100084, P.R. China

${ }^{4}$ Interdisciplinary Research Centre in Biomedical Materials, COMSATS Institute of Information Technology, Lahore-54000, Pakistan

*Corresponding author: Fax: +92 42 99230998; Tel: +92 42 99230436; E-mail: tajamalhussain.chem@ @ pu.edu.pk

Reverse micro emulsion is often used for the synthesis of metal nanoparticles. Such prepared metallic nanoparticles may be used as catalyst for oxidation of methanol occurring in fuel cell. Nickel electrodes were modified by electro chemical deposition of the nickel nanoparticles. In-continuous deposition was occurred on using reverse micro emulsion. This problem was addressed on having the same in the presence of magnetic field. In fact, Lorentz force started playing role in terms of large mass transfer rate when magnetic field perpendicular to the direction of current was applied. Surface analysis of the both types of the modified electrode was done with the help scanning electron microscopy. Electro chemical and catalytic studies of both modified electrodes were done. Results of these studies encouraged the employment of the magnetic field during the electro chemical deposition of the metals.

Keywords: Magnetic field, Magneto-hydrodynamic effect, Reverses micro emulsion, Electrochemical deposition.

\section{INTRODUCTION}

Microemulsions are a distinctive class of thermodynamically stable, optically clear and homogeneous, at macro level, medium. They have low viscosity and spontaneously formed solution phase. Generally, microemulsions are composed of aqueous, organic solvents and anionic, cationic or neutral surfactants ${ }^{1-4}$. When dealing on micro level an emulsion is a heterogeneous in nature since microemulsion composed of droplets of either oil in water $(\mathrm{O} / \mathrm{W})$ or water dispersed in oil $(\mathrm{W} / \mathrm{O})^{5}$. Water-in-oil micro emulsion also known as modified reverse micro emulsion (RME) contains water pools stabilized by surfactant disperse in continuous oil phase ${ }^{6-8}$. Micro emulsions are famous and have remarkable features due to their unique characteristics like micro-heterogeneity and the macro-homogeneity. Because of this interesting feature, micro emulsions can be conveniently employed to develop materials widely used in various fields like catalysis, sensors, model membrane systems, solubilization media, drug delivery, etc. ${ }^{9-13}$. Besides the above said characteristics, one of the important application of microemulsions is the use as template for synthesis of nanoparticles of size ranging from 20 to $400 \mathrm{~nm}$. In such synthesis, the micro emulsion phase acts as an inert medium to prevent the coalescence or agglomeration of nanoparticles ${ }^{14-17}$ during the growth. The size and morphology of the resultant nanoparticles can be controlled by adjusting the size of nano-water pools ${ }^{18-19}$. There is a considerable interest in the fabrication of high surface area metal nanostructures as thin films for a wide variety of applications including energy storage (super capacitors), energy conversion (fuel cells), separations (electrochemical chromatography), sensors and catalysis ${ }^{20-23}$. Besides the other chemical synthesis method of nanoparticles in reverse micro emulsion ${ }^{24,25}$, electrochemical technique is an effective one to prepare nano-scaled materials as it is a straight forward method for the production of functional and productive thin films as well as nanoparticles ${ }^{26,27}$. Along the different advan$\operatorname{tages}^{28}$, there are few problems related to the use of reverse micro emulsion as template for the synthesis of the nanoparticles particularly when synthesis is being carried out electrochemically. This is because electrode process at electrode/ microemuslion interface is quite different than that of electrode/ aqueous interface ${ }^{29,30}$. Since electrochemical reactions in reverse micro emulsion are charge transfer controlled rather than diffusion controlled. So limited mass transformation occurs as only the metal ions present in the adsorbed water molecule at the surface of the electrode are getting deposited ${ }^{31}$. Non-aqueous part of the emulsion in contact with the surface does not take part in the deposition process. Therefore, all this 
become the reason of in continuous deposition ${ }^{29}$. One of the important features of the electro-deposition in the presence of magnetic field, perpendicular to the flow of the current, is the observation of the large mass transfer rate. As a result comparatively dense surface is formed ${ }^{32-33}$. This effect of the magnetic field is known as the magneto-hydrodynamic (MHD) effect. Magneto-hydrodynamic effect actually increases the value of limiting current. The origin of this effect lies in the Lorentz force, $F_{L}=\mathrm{i} \times \mathrm{B}$, where $\mathrm{i}$ is the current density and $\mathrm{B}$ is the magnetic field. Detailed mechanism of the deposition is given by Ebadi et al..$^{33}$. In the present work, reverse micro emulsion is used as template for the modification of nickel electrodes by electrochemical deposition of the nanoparticles of the same metals when no magnetic field is applied and in the presence of magnetic field. Surface of the modified electrodes are analyzed by scanning electron microscopy (SEM). Surface studies are also done by cyclic voltammeter. Efficiency of the electrodes in terms of electro oxidation of the ethanol and methanol in basic media is also investigated.

\section{EXPERIMENTAL}

p-Octyl polyethylene glycol phenyl ether (Triton X-100), non-ionic surfactant, $n$-hexanol (Fluka) and $n$-hexane (Fluka), co-surfactants, nickel sulfate $(\mathrm{BDH})$, hydrochloric acid (Sigma-Aldrich), sodium hydroxide (Fluka), methanol (SigmaAldrich) and ethanol (Sigma-Aldrich) were used as obtained without any specific purification or treatment. All chemical reagents used were of analytical grade. Doubly distilled water was used for solution preparations.

Synthesis of solution using reverse micro emulsion: $n$ Hexane and $n$-hexanol, as the oil phase, are mixed in 1:1 volume ratio and Triton X-100, half of the volume of $n$-hexane, was added in it. Mole ratio of water to surfactant was kept 10.4. The water phase contained $0.3 \mathrm{~mol} / \mathrm{L}$ of $\mathrm{NiSO}_{4} .7 \mathrm{H}_{2} \mathrm{O}$ and $9.5 \mathrm{~mol} / \mathrm{L}$ of $\mathrm{HCl}$. Microemulsion is not very good conductor $^{30}$, so this is why $9.5 \mathrm{~mol} / \mathrm{L} \mathrm{HCl}$ is added to enhance its conductivity. Microemulsion phase acts as a medium for the electrodeposition of nickel. So the stability of prepared microemulsion is very significant. In order to check the stability, first reverse micro emulsion was prepared containing only water as an aqueous phase and centrifuged with 6,000 rpm at $25{ }^{\circ} \mathrm{C}$ for $15 \mathrm{~min}$. Then reverse micro emulsion containing 9.5 $\mathrm{M} \mathrm{HCl}$ solution as an aqueous phase was prepared and centrifuged. For both cases after centrifuge, reverse micro emulsion remained transparent and no phase separation took place. The result demonstrates that reverse micro emulsion containing highly acidic aqueous phase is also quite stable. Then reverse micro emulsion containing $9.5 \mathrm{M} \mathrm{HCl}$ and $0.3 \mathrm{M}$ $\mathrm{Ni}_{2} \mathrm{SO}_{4} .7 \mathrm{H}_{2} \mathrm{O}$ were also processed through the above mentioned procedure. The reverse micro emulsion containing $\mathrm{Ni}$ salt solutions were stable before and after electrodeposition for months.

Electro deposition: Electro deposition of Ni nano-particles was carried out in a two-electrode cell using reverse micro emulsion as template. Cathode was nickel electrode having diameter $5 \mathrm{~mm}$. Glassy carbon electrode was used as anode. Before electro deposition activation treatment of the nickel electrode was carried out by etching in a mixture of $\mathrm{H}_{2} \mathrm{SO}_{4}$,
$\mathrm{HNO}_{3}, \mathrm{HCl}$ and chromic acid for $30 \mathrm{sec}$. Electrode was then rinsed in distilled water and air dried before every experiment. The inter-electrode distance was $2 \mathrm{~cm}$. All the experiments were conducted at $22{ }^{\circ} \mathrm{C}$. The electro deposition was carried out at a potential difference of $4.50 \pm 0.02 \mathrm{~V}$ for $15 \mathrm{~min}$ in two electrode system. To have electrodeposition done when magnetic field is present, deposition cell put in between two poles of the home made electrical magnet of strength of $0.3 \mathrm{~T}$.

Surface morphology of the formed surface is characterized by Hitachi SU-1500 Scanning Electron Microscopy (SEM). Electrochemical analysis was done with Cyclic Voltammetry (CV) Eco Chemie Autolab PGSTST 12 potentiostat/galvanostat Netherlands, with electrochemical software pakage GPES 4.9. Catalytic activity of the electrode modified in the presence and absence of magnetic field is carried out by electro oxidation of methanol and ethanol in basic environment. Modified nickel electrode is taken as anode and graphite electrode as cathode for catalytic studies.

\section{RESULTS AND DISCUSSION}

Surface analysis by SEM: SEM images of the surface of the modified electrodes are given in the Figs. 1 and 2, SEM image given in Fig. 1, is for the particles deposited in the absence of magnetic field with $0.3 \mathrm{M}$ concentration of $\mathrm{Ni}$ ions in solution while the SEM image given in the Fig. 2 is for the particles deposited in the presence of magnetic field of strength 0.3 T. On comparing the SEM images, it is clear that extent of the deposition is different when there is magnetic field applied as compared to when no magnetic field is applied. Comparatively dense deposition takes place due to the magnetic field which indicates that Lorentz force plays the role and large mass transfer rate is observed. In present case almost $5 \%$ large current is observed when process of deposition taken place in the presence of magnetic field than when no magnetic field is there keeping the applied potential and time of the deposition same along the other factors. Same enhanced current is observed at another place ${ }^{34}$. It also observed that presence of the magnetic field do not cause any phase separation in reverse micro emulsion after the completion of the experiment.

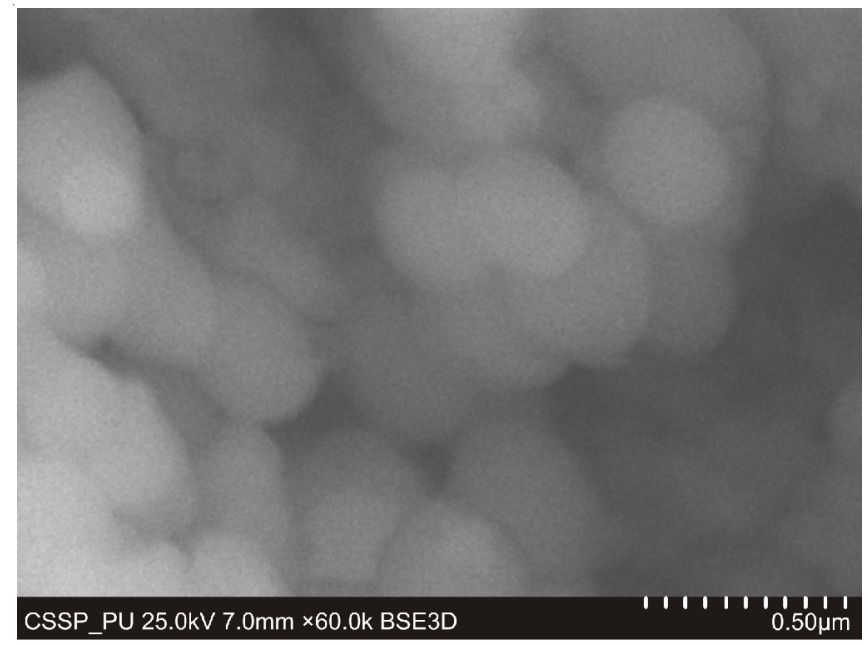

Fig. 1. SEM images of the nickel surface electrodeposited in reverse micro emulsion as template without magnetic field 


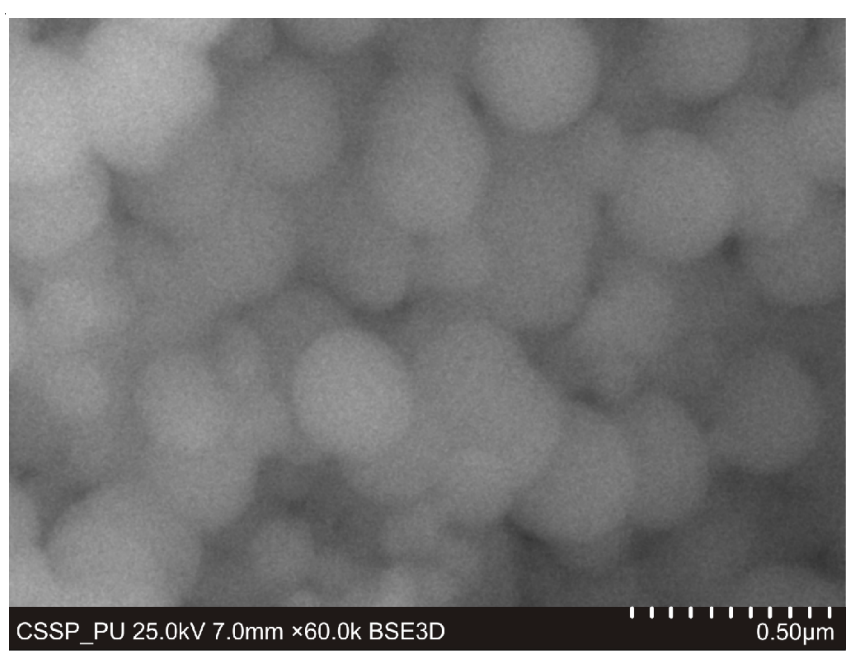

Fig. 2. SEM images of the nickel surface electrodeposited in reverse micro emulsion as template in the presence of magnetic field

Electrochemical study by cyclic voltammetry: Study of electro active surface area of Ni electrodes modified both in the presence and absence of magnetic field from reverse micro emulsion with $\mathrm{Ni}$ ions concentration of $0.3 \mathrm{M}$, is done by the amount of $\mathrm{Ni}$ hydroxide formed and reduced during cyclic voltammetry in $1 \mathrm{M} \mathrm{NaOH}$. Fig. 3 shows the cyclic voltammogram at $50 \mathrm{~m} \mathrm{Vs}^{-1}$ of deposit produced in the absence and presence of magnetic field in a degassed $1 \mathrm{M} \mathrm{NaOH}$. It is obvious from the voltammograms that in basic medium the electrode surface becomes covered with oxide/hydrous oxides $\left[\mathrm{Ni}(\mathrm{OH})_{2}\right.$ or $\left.\mathrm{NiOOH}\right]$ at above $0.0 \mathrm{~V}^{35}$. The monolayer of $\alpha-\mathrm{Ni}(\mathrm{OH})_{2}$ formed during the anodic scan is stripped off during the reverse scan beyond $-0.6 \mathrm{~V}$ versus $\mathrm{SCE}$ as seen from the oxide stripping peak ${ }^{36-37}$. However the $\mathrm{CV}$ of Ni coating deposited in the presence of magnetic field shows a rather drawn out oxide formation region signifying an increase in the true surface area and therefore the overall charge associated with the process. Hydrogen evolution can be observed at potential -0.6 to $-1.2 \mathrm{~V}$ during the negative scan. Comparing the curves, it can be deduced that hydrogen reduction peak current on $\mathrm{Ni}$

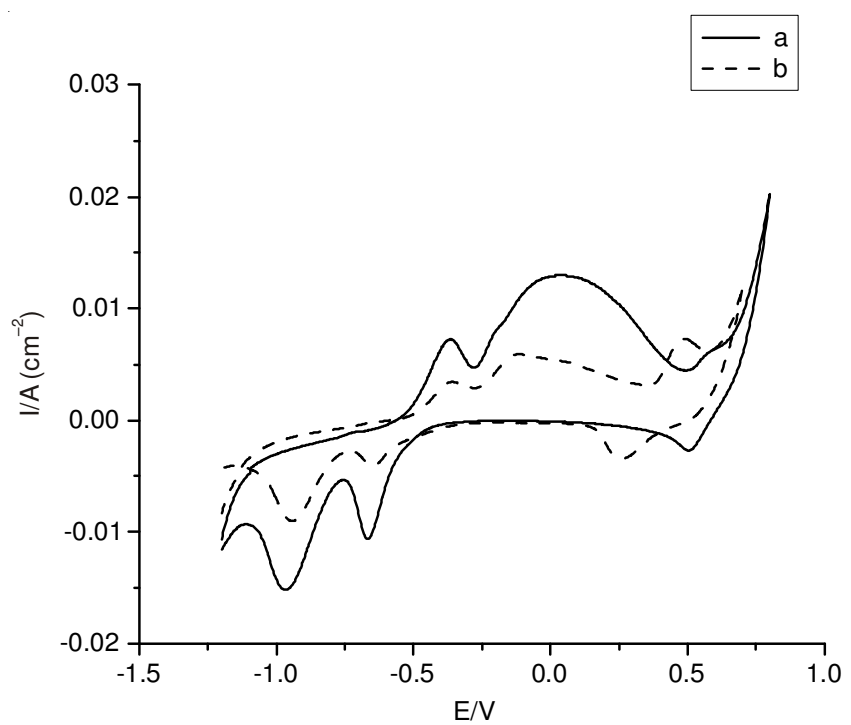

Fig. 3. Cyclic voltammograms at $50 \mathrm{mVs}^{-1}$ scan rate recorded in $1 \mathrm{M} \mathrm{NaOH}$ of the nickel surface electrodeposited a) in the presence of magnetic field $b$ ) without magnetic field (E vs. SCE) nano-particles plated in the presence of magnetic field is higher than that on Ni plated in the absence of magnetic field. 514 $\mu \mathrm{C} / \mathrm{cm}^{-2}$ is the amount of charge correspond to the formation of monolayer of $\alpha-\mathrm{Ni}(\mathrm{OH})_{2}{ }^{38}$.

Electro oxidation of methanol and ethanol: Electro oxidation of methanol and ethanol in aqueous solution of 0.1 $\mathrm{M} \mathrm{NaOH}$ is being done using anode as modified electrodes in the presence and absence of magnetic field and graphite electrode as cathode as reported in literature ${ }^{39}$. Results are given in terms of plots of applied voltage and current density in the Fig. 4. It is cleared from the graphs that for oxidation of both of the alcohols, current density is larger for electrode modified in the presence of the magnetic field as compared to that of in the absence of magnetic field, this goes to generation of large surface area in the presence of magnetic field.

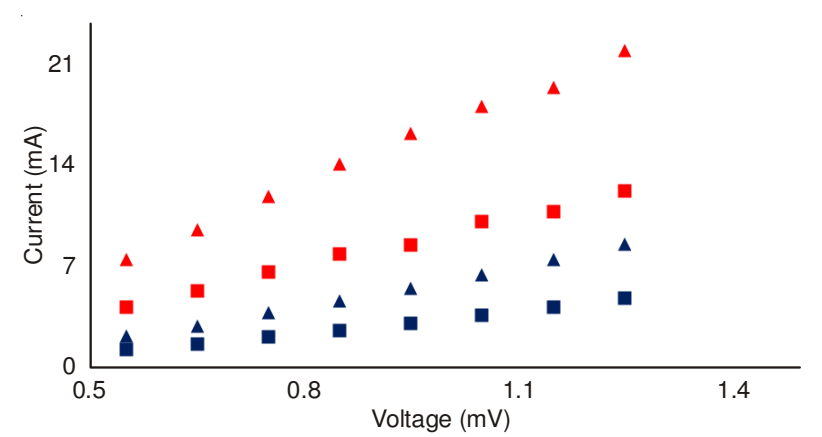

Fig. 4. Electroxidation of ( $\square$ ) methanol and ( $\mathbf{\square})$ ethanol by using electrode modified without magnetic field and $(\boldsymbol{\Delta})$ methanol and $(\boldsymbol{\Delta})$ ethanol by using electrode modified when magnetic field is present

\section{Conclusion}

Use of reverse microemulsion as a template for the electro deposition of the nanoparticles is very convenient. Through present study, it is clear that reverse microemulsion can be made more effective for the synthesis of the nanoparticles electrochemically. As a result of magneto-hydrodynamic effect, serious problem of incontinous deposition, when reverse microemulsion is used as template, can be solved out by employing magnetic field perpendicular to the direction of current during the deposition as confirmed my SEM images. Moreover, electro chemical studies indicate the large surface area of electrode modified in the presence of magnetic field.

\section{ACKNOWLEDGEMENTS}

Tajamal Hussain is highly thankful to Higher Education Commission, Pakistan and University of the Punjab, Lahore, Pakistan for providing funds to carry out this work and Prof. Dr. Shahzad Naseem, Director, Centre for Solid State Physics, University of the Punjab, Lahore for providing facility of SEM analysis.

\section{REFERENCES}

1. T. Hellweg, Curr. Opin. Colloid Interface Sci., 7, 50 (2002).

2. R.A. Mackay and J. Texter, Electrochemistry in Colloids and Dispersions, VCH, New York (1992).

3. J.F. Rusling, Acc. Chem. Res., 24, 75 (1991).

4. R.A. Mackay, Colloids Surf. A, 82, 1 (1994).

5. A.W. Adamson, Physical Chemistry of Surfaces, John Wiley and Sons, New York (1976). 
6. C. Fu, H. Zhou, H. Wu, J. Chen and Y. Kuang, Colloid Polym. Sci., 286, 1499 (2008).

7. C.H. Lu, H.H. Chang, C. Yen and J. Tamkang, Sci. Eng., 7, 199 (2004).

8. H. Li, J. Liu, S. Xie, M. Qiao, W. Dai and H. Li, J. Catal., 259, 104 (2008).

9. T.C. Franklin, S. Mathew and K.L. Mittal, Surfactant in Solutions, Plenum, New York (1989).

10. J.H. Fendler, Membrane Mimetic Chemistry, Wiley-Interscience, New York (1982).

11. E. Pelizzetti and E. Pramauro, Anal. Chim. Acta, 169, 1 (1985).

12. P.L. Luisi, M. Giomini, M.P. Pileni and B.H. Robinson, Biochim. Biophys. Acta, 947, 209 (1988).

13. K. Shinoda and B. Lindman, Langmuir, 3, 135 (1987).

14. A. Manna, T. Imae, T. Yogo, K. Aoi and M. Okazaki, J. Colloid Interf. Sci., 256, 297 (2002).

15. M.P. Pileni, Nat. Mater., 2, 145 (2003).

16. H. Zhou, C. Peng, S. Jiao, W. Zeng, J. Chen and Y. Kuang, Electrochem. Commun., 8, 1142 (2006).

17. C. Destrée, J. Ghijsen and J. B.Nagy, Langmuir, 23, 1965 (2007).

18. L. Xiong and A. Manthiram, Solid State Ion., 176, 385 (2005).

19. X. Zhang and K.Y. Chan, Chem. Mater., 15, 451 (2003).

20. R. Campbell, M.G. Bakker, C. Treiner and J. Chevalet, J. Porous Mater. 11, 63 (2004).

21. R. Campbell, M.G. Bakker, G. Havrilla, V. Montoya, E. Kenik and M. Shamsuzzoha, Micropor. Mesopor. Mater., 97, 114 (2006).

22. A. Seghiouer, J. Chevalet, A. Barhoun and F. Lantelme, J. Electroanal. Chem., 442, 113 (1998).

23. J.P. Hsu and A. Nacu, J. Colloid Interf. Sci., 259, 374 (2003).
24. W. Zhang, X. Qiao and J. Chen, Colloids Surf., A, 299, 22 (2007).

25. W. Zhang, X. Qiao and J. Chen, Chem. Phys., 330, 495 (2006).

26. L. Huang, H. Wang, Z. Wang, A. Mitra, K.N. Bozhilov and Y.S. Yan, Adv. Mater., 14, 61 (2002).

27. Y.X. Pang and X. Bao, J. Mater. Chem., 12, 3699 (2002).

28. C. Fu, H. Zhou, D. Xie, L. Sun, Y. Yin, J. Chen and Y. Kuang, Colloid Polym. Sci., 288, 1097 (2010).

29. H. Zhou, C. Peng, C. Fu, J. An, H. Zou, Y. Wang, Y. Xu and Y. Kuang, J. Cent. South Univ. Technol., 17, 40 (2010).

30. H. Zhou, C. Peng, S. Jiao, W. Zeng, J. Chen and Y. Kuang, Electrochem. Commun., 8, 1142 (2006).

31. V. Ganesh and V. Lakshminarayanan, J. Colloid Interf. Sci., 349, 300 (2010).

32. A. Bund, A. Ispas and G. Mutschke, Sci. Technol. Adv. Mater., 9, 024208 (2008).

33. M. Ebadi, W.J. Basirun and Y. Alias, J. Chem. Sci., 122, 279 (2010).

34. M. Ebadi, W.J. Basirun, Y. Alias and M. Mahmoudian, Chem. Cent. J., 4, 14 (2010).

35. V. Ganesh and V. Lakshminarayanan, Electrochim. Acta, 49, 3561 (2004).

36. I.J. Brown and S. Sotiropoulos, Electrochim. Acta, 46, 2711 (2001).

37. I.J. Brown and S. Sotiropoulos, J. Appl. Electrochem., 30, 107 (2000).

38. V. Ganesh and V. Lakshminarayanan, J. Colloid Interf. Sci., 349, 300 (2010).

39. A.T. Shah, M.I. Din, U. Farooq, M.T.Z. Butt, M. Athar, M.A. Chaudhary, M.N. Ahmad and M.L. Mirza, Colloids Surf. A, 405, 19 (2012). 